\section{Surfing for light waves}

\section{A. R. Bell}

THE diversity of nonlinear interactions involving waves in plasmas is both a fascination and a frustration to plasma physicists. Often the interactions arise unexpectedly to frustrate attempts to design useful plasma devices, but the very wealth of interactions gives great scope for inventive ideas. The latest ingenious idea springs from the University of California at Los Angeles where S. C. Wilks, J. M. Dawson and co-workers have recently suggested that it may be possible to 'accelerate' photons in a plasma (Phys. Rev. Lett. 62, 2600-2603; 1989). By this they Plasma wave Light

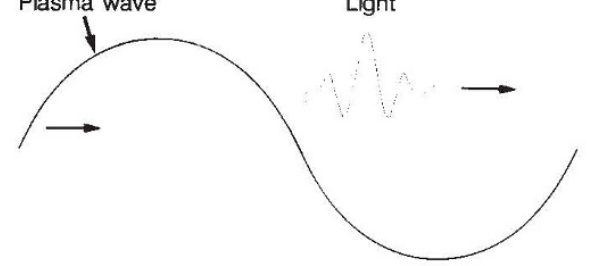

Schematic of the photon accelerator, showing the electron plasma wave, with the wavefront moving close to the speed of light and boosting the frequency of the light pulse.

mean that photons initially with low energy can be changed into high-energy photons as they pass through a suitably excited plasma.

Behind the concept is the hope of producing a high-frequency, coherent electromagnetic wave by upshifting a lowfrequency coherent wave - an alternative to direct laser action at the higher frequency. The idea presents an enticing prospect. Coherent high-energy radiation is not easily produced, especially when $\mathrm{X}$-ray energies are approached. The proposed scheme is similar to those currently being investigated for accelerating charged particles - for example the plasma beatwave accelerator (PBWA). Indeed, Dawson was, with T. Tajima, one of the originators of the beat-wave and related ideas (Phys. Rev. Lett. 43, 267-270; 1979).

In either scheme, a large-amplitude wave is generated in an ionized plasma by intense laser beams or by an energetic particle beam. The wave consists of a sinusoidal variation in electron density which moves with a phase velocity slightly less than the speed of light.

For simplicity, view the process in the reference frame moving with the plasma wave. In this frame the sinusoidal density variation is stationary. In the photon accelerator, a short pulse of electromagnetic radiation (short compared with the wavelength of the plasma wave) can be trapped in one of the low-density troughs of the wave, because electromagnetic radiation of a given frequency cannot propagate above a certain density (the critical density). The density trough can be seen as behaving like a potential well for the photons comprising the electromagnetic pulse. The group velocity of an electromagnetic wave propagating up a density ramp reduces as the density increases, until it becomes zero at the critical density where it is reflected down 'the density gradient. A pulse initially placed at the bottom of the trough and travelling backwards with respect to the wave will climb the wall of the trough, reverse its direction when it reaches the critical density and returns to its original position with its original frequency and energy. Its group velocity is unchanged in magnitude but reversed in direction.

Transforming back into the laboratory frame, we see that because of the change of direction, the magnitude of the laboratory group velocity has in fact increased: the pulse starts at the bottom of the trough, moving in the same direction as the plasma wave but at a slightly lower velocity (a suitable choice of light frequency gives the required group velocity). The pulse initially drops back relative to the wave, but then accelerates to a velocity higher than that of the plasma wave, eventually returning to the bottom of the trough with an increased group velocity. Because the group velocity has increased, so have its frequency and photon energy - the photons have been accelerated.

The analysis by Wilks et al. shows that the basic scheme can increase the electromagnetic frequency by a factor of around 5, although this requires a plasma wave in which the excursion of the electon density from its mean is half the mean density. This amplitude is much larger than that of any coherent plasma wave created so far by proponents of the PBWA, but it is not impossible given the rapid developments in this field. To obtain a larger frequency upshift or to manage with less extreme plasma waves, Wilks et al. suggest steadily raising the plasma density along the length of the accelerator.

Many problems still need to be addressed. For example, a suitable means of driving a plasma wave with the required amplitude, coherence and phase velocity needs further investigation. The ultimate efficiency of the system is uncertain. Undesirable wave-wave interactions could compete with those desired for photon acceleration. And problems could arise when the present one-dimensional analysis is replaced by a full two- or threedimensional one. The proof of the theoretical pudding will be in the experimental realization.

A. R. Bell is in the Blackett Laboratory, Imperial College of Science, Technology and Medicine, Prince Consort Street, London SW7 2BZ, UK.

\section{Bubbling upwards}

LAST week Daedalus invented a solution for blowing really robust soap bubbles. It contains big closed-loop polymer molecules: any small puncture is certain to be surrounded by at least one loop molecule. So it cannot spread and is soon healed by brownian motion. Invulnerable to the usual mode of failure, a 'Hyberbubble' is a wonderfully light and robust structure, rich with technological promise.

Daedalus is directing this promise firmly upwards. He points out that a traditional balloon is only partly filled with gas before its release. As long as its envelope is slack, the pressure inside and outside are equal and the lift of the balloon remains constant. But as it rises through the thinning air, it expands steadily and ultimately fills taut. The balloon has reached its ceiling: any further ascent reduces its lift.

But a Hyperbubble would behave quite differently. Such a stable soap bubble would simply expand indefinitely as it rose and would pull taut only when it had thinned to a perfect monolayer. Daedalus calculates that a hydrogen-filled Hyperbubble with an initial radius of a mere metre or so, and an initial film thickness of $0.1 \mathrm{~mm}$, could lift itself from sea level and reach about $160 \mathrm{~km}$ altitude. In practice, of course, the bubble would have the usual $0.001 \mathrm{~mm}$ soap-film thickness even at ground level, but could carry the extra liquid in a reservoir to top-up the thinning film as it expanded at higher altitudes.

Attaching a payload to a big liquidskinned balloon poses intriguing problems. Daedalus hopes that a complex corded harness, light and fairly flexible, can be given such an extended line of wetted contact with the bubble that the total surface tension along the line will support a few kilograms of instruments. A spacegoing Hyperbubble cannot contain water, which would evaporate rapidly in the low pressure. Liquid polymers, high-molecularweight detergents and other novel soapfilm components are being investigated. But ultimately Hyperbubbles should lift research equipment up to $100-200 \mathrm{~km}$ amazingly simple, cheaply and reliably.

At operating altitude a Hyperbubble will be a vast attenuated object drifting before whatever vacuous molecular wind there is up there. Though immune to punctures from micrometerorites, it will still slowly lose gas by diffusion, and gradually descend. In the process it will slowly shrink and thicken, at each stage forming an effective parachute for the local atmospheric density. Cosmic-ray film packs, survey cameras, magenetometers, all will be lifted gently and cheaply into near space and as gently returned. The robustness and reliability of a Hyperbubble would only really be overwhelmed by collision with an orbiting satellite.
David Jones 